Commentary

\title{
Could stress granules be involved in age-related diseases?
}

\author{
Imed-Eddine Gallouzi
}

\section{McGill University, Department of Biochemistry, Rosalind and Morris Goodman Cancer Center, Montreal, Quebec, Canada}

Running title: The impact of stress on senescent cells

Key words: senescence, stress granules, oxidative stress

Correspondence: Imed-Eddine Gallouzi, PhD, McGill University, Department of Biochemistry, McIntyre Building, room 904, 3655 Promenade Sir William Osler, Montreal, Quebec, H3G 1Y6, Canada

Received: 09/10/09; accepted: 09/19/09; published on line: 09/21/09

E-mail: imed.gallouzi@mcgill.ca

Copyright: (C) 2009 Gallouzi. This is an open-access article distributed under the terms of the Creative Commons Attribution License, which permits unrestricted use, distribution, and reproduction in any medium, provided the original author and source are credited

Cellular senescence, an irreversible cell-cycle arrest, acts as a safeguard program that limits the proliferative capacity of cells when exposed to endogenous or exogenous stress signals [1, 2]. However, the senescence phenotype is also considered as a sign that the life span of a cell has reached its end. Indeed, in the early 1960s, Hayflick and Moorhead showed that despite the maintenance of optimal culturing conditions for a long period of time, normal cells do not proliferate forever [35]. For example, they observed that normal human diploid fibroblasts have a limited proliferation capacity and after a finite number of population doubling, they stop dividing and enter senescence. The occurrence of replicative senescence has been demonstrated for most cell types, with a few relevant exceptions including embryonic germ cells [6]. In human cells, the primary cause of cellular senescence appears to be the progressive shortening of telomeres, which are DNA structures at the end of eukaryotic chromosomes [7-9]. Senescence can also be induced by non-telomeric signals, termed "premature" or "accelerated" senescence [10]. Senescence-inducing signals, such as DNA-damage response (DDR) and oxidative stress (OS), usually engage either the p53 or the cyclin-dependent kinase inhibitor p16 pathway [11-14]. Active p53 establishes senescence, in part, by inducing the expression of the cyclin-dependent kinase inhibitor $\mathrm{p} 21^{\mathrm{cip}}$, which suppresses the phosphorylation of the retinoblastoma protein pRB, leading to its inactivation [15].

The importance of the senescence phenotype is underscored by the fact that this condition could trigger two opposite outcomes. Due to its antiproliferative effect, senescence is activated to prevent further growth of transformed or sick cells that are subsequently eliminated by the immune system [10, 16]. This effect has mainly been observed in young organisms, and as such senescence is considered a natural tumor suppressor mechanism [16-19]. On the other hand, the situation seems to change in aged individuals. Indeed, several studies have suggested that as we age, many senescent cells escape the immune system and end up accumulating in different tissues for a long period of time, which correlates with age-related diseases such as cancer $[1,16,20]$. This functional dichotomy of the senescence phenotype raises questions such as how and why the same conditions could lead to opposite outcomes depending on age. While the answers to these questions are still elusive, it is possible that the switch of senescent cells from being a natural break of tumor growth to becoming promoters of malignancy occurs over a long period of time as a consequence of repeated exposure to stress during the life-span of an organism. Since senescent cells remain metabolically active [7, 19], these stresses could cause dramatic changes in the expression pattern of key genes which could explain how and why as we age senescent cells switch their function to become promoters of tumor growth. Although the expression patterns and the activities of the genes involved in promoting and maintaining the senescence state are well studied, very little is known about the effect stress could have on their expression when the cell are fully senescent. 
To address the questions asked above we assessed how senescent cells, incapable of further division, react to extracellular assaults. Our recent work [21] clearly indicated that in response to stress, senescent cells activate mechanisms that are similar to those seen in exponentially growing cells. We observed that fully senescent cells exposed to stresses such as oxidative stress (OS) or heat shock are able to form bonafide stress granules (SGs) [21]. Originally, SGs were identified as cytoplasmic RNA granules that form in mammalian cells upon exposure to various stresses [2224]. SG assembly represents one of the main prosurvival mechanisms through which cells cope with environmental assaults by helping them reprogram mRNA metabolism and repair stress-induced damage. We observed that when fully senescent human fibrobalsts exposed to either heat shock, or to arsenate (AS), a well-known inducer of OS, a much higher number of SGs form than in exponentially growing cells [21]. Since it is known that senescent cells have a decreased capacity to adapt to environmental stresses $[25,26]$, we assessed the impact of this high number of SGs on their ability to recover from these assaults. We observed that upon switching cells to AS-free media, SG disassembly in fully senescent cells occurs at a slower rate than in cells at earlier stages of the senescence process. Recent experiments performed in my laboratory support this observation and showed that in fully senescent cells there is a small but reproducible decrease in the expression levels of the heat shock protein 70 (HSP70), which is one of the key players involved in cell recovery from a variety of stresses [27, 28] (Figure 1). This already suggested that while senescent cells maintained the ability to form SGs in response to stress, their recovery process was affected. This result is consistent with previous in vitro and in vivo studies showing that the expression of HSP70 decreases in senescent cells exposed to stresses such as heat shock [29, 30]. Hence, together these data argue that the slow recovery rate observed in fully senescent cells [21] could be explained by the delayed expression of HSP70 protein. While SGs are entities used as a protective mechanism under stressful conditions, the fact that they take more time to disassemble in senescent cells upon stress removal could indicate a delay in the synthesis of many vital proteins needed for the maintenance of the senescence status.

It is well accepted that in exponentially growing cells SGs recruit a variety of mRNA not only for protection from decay and sorting purposes but also to block their translation during cell exposure to stress [32]. As soon as the stress is relieved, however, SG disassemble and translation resumes. Hence, we investigated whether this could also be the case in senescent cells. Our data
A
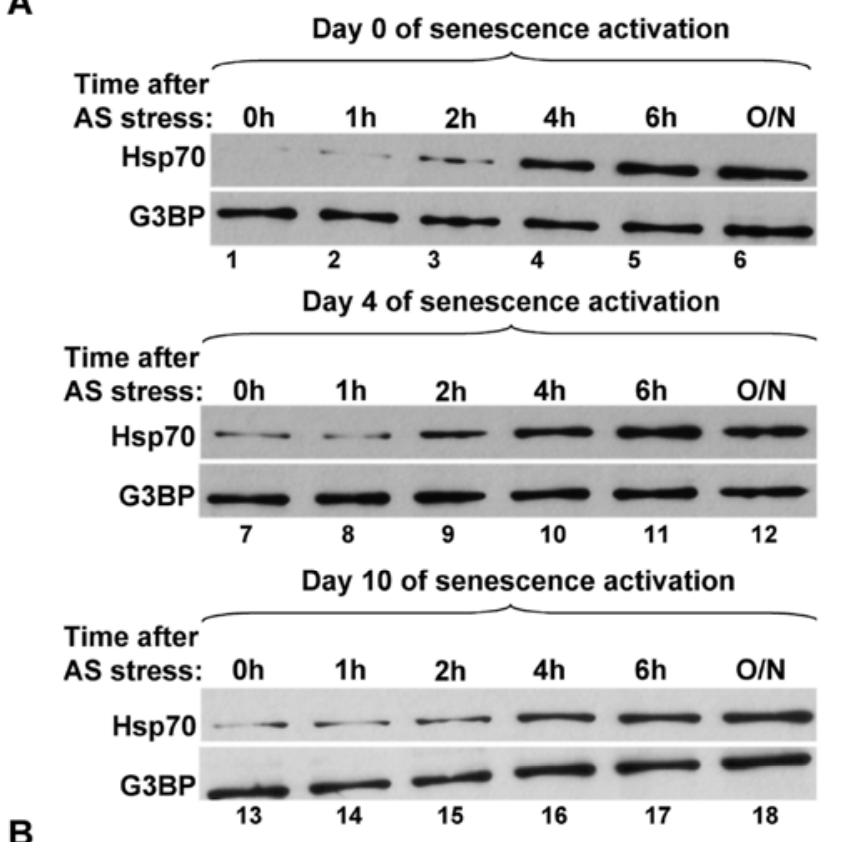

B

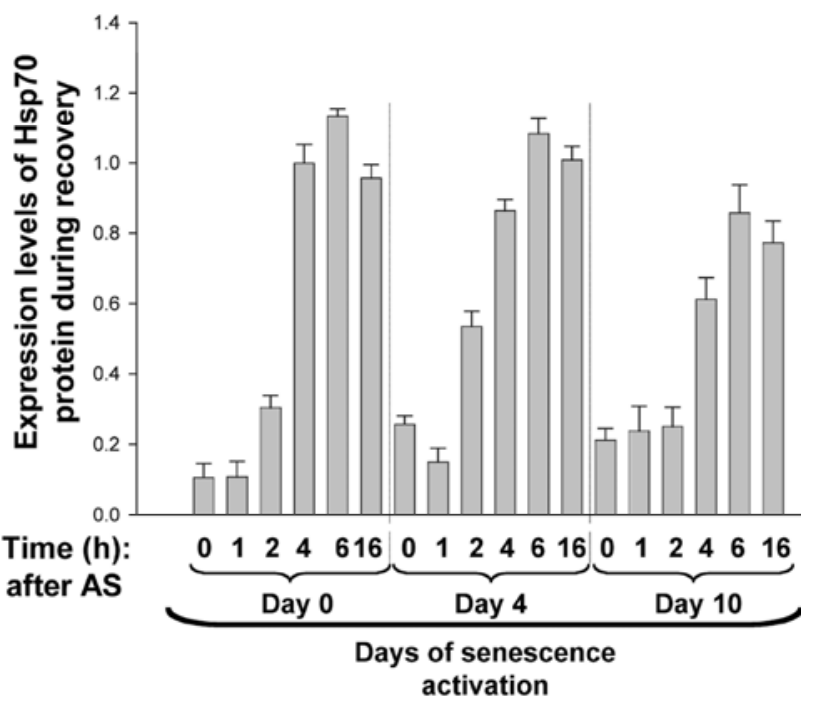

Figure 1. The upregulation of Hsp70 expression correlates with SG disassembly during the recovery from AS stress of proliferative and senescent cells. (A-B) Expression levels of Hsp70 protein after the removal of AS stress. (A) Proliferative and senescent IDH4 cells were incubated with arsenite $(0.5 \mathrm{mM})$ for 30 $\mathrm{min}$. Cells were subsequently washed twice with PBS, replenished with fresh media and incubated for various periods of time at $37^{\circ} \mathrm{C}$. Total cell extracts prepared from these cells were then used for Western blots analysis with antibodies specific to Hsp70 and G3BP (used as the loading control). Representative western blots of three independent experiments are shown. (B) The bar graphs represent the expression level of Hsp70 protein in each time point normalized to the expression levels of the loading control G3BP. The intensity of the signal in each lane was measured using ImageQuant software. Each bar graph represents the ratio of Hsp70 over G3BP for each time point. The histogram presents the results from (A) as a mean +/- SEM (error bars), from three independent experiments. 
indicate that the effect of OS-induced SGs on the expression of the $\mathrm{p} 21^{\text {cip }}$ mRNAs is dependent on the senescence stage of the cell [21]. We showed that although the $\mathrm{p} 21^{\mathrm{cip}}$ mRNAs colocalizes with SGs in both early and fully senescent cells, the synthesis of $\mathrm{p} 21^{\text {cip }}$ protein was rapidly shut off only in fully senescent cells. The fact that these cells were treated with a sub-lethal dose of AS for only a short period of time (30 min), indicates that the events leading to the translation inhibition of $\mathrm{p} 21^{\mathrm{cip}}$ mRNA are triggered quickly and correlate with the assembly of SGs. This, however, does not explain why p $21^{\text {cip }}$ translation is not affected in cells at earlier stages of the senescence process despite the fact that in these cells the p21 ${ }^{\text {cip }}$ message is also rapidly recruited to SGs. Surprisingly, our data raise the possibility that senescent and normally growing cells use different molecular mechanism to assemble SGs in response to OS. We observed that in both early and fully senescent cells the phosphorylation of eIF2 $\alpha$, a key factor in AS-induced SG formation [33], is significantly reduced [21]. This result suggests that during the senescence process OSinduced SG formation switches from an eIF2 $\alpha$ phosphorylation-dependent mechanism to a process that is independent of this posttranslational modification. Work from several groups including ours, have demonstrated the existence of an eIF2 $\alpha$ phosphorylation-independent mechanism for SG assembly. Indeed, cells exposed to Pateamine A or hippuristanol, two well-known inhibitors of the eukaryotic translation initiation factor A (eIF4A) form SGs in an eIF2 $\alpha$ phosphorylation-independent manner [34, 35]. Although this or similar mechanisms could explain SGs formation in senescent cells exposed to OS, this possibility needs to be tested experimentally. Defining the mechanisms by which SGs assemble in senescent cells could open the door to screen for chemical inhibitors/activators that modulate SG formation in these cells. This could in turn provide tools to design new strategies that prevent senescent cell from promoting malignancy.

In summary, delineating the functional relevance of SGs in senescent cells exposed to a variety of extracellular drugs could be relevant to the treatment of age-related diseases such as cancer. Indeed, many chemotherapeutic agents are used due to their ability to trigger senescence in cancer cells. Though a growing number of small molecules that induce irreversible cell cycle arrest in malignant cells have been recently developed, improvement of cancer treatment is limited, underscoring the need for identifying the mechanisms by which these treatments could modify the behavior of senescent cells [10, 20, 36-38]. In fact, in some cases, malignant cells exposed to repeated treatments with these molecules may

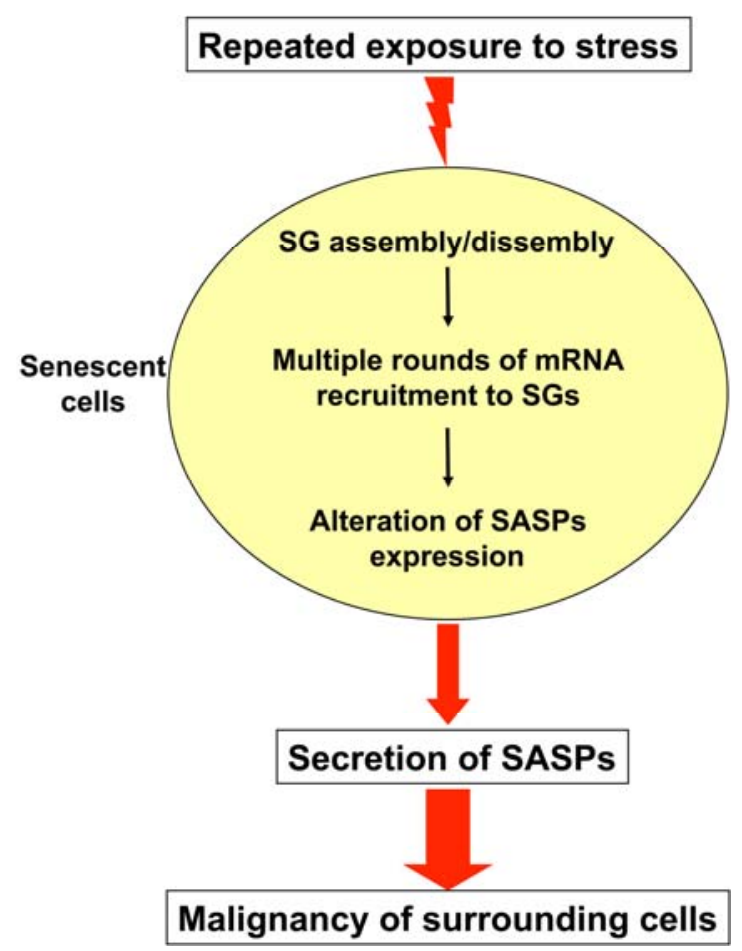

Figure 2. Working model of how repeated exposure to stress could change the pattern of mRNA expression in senescent cells. In response to stresses such as oxidative stress and heat shock senescence cells form a high number of stress granules (SGs). These SGs recruit several mRNAs leading to their translation inhibition. During its lifespan, a living organism is exposed repeatedly to a variety of stresses. This could trigger multiple cycles of SGs assembly/disassembly which in turn change the expression pattern of mRNAs encoding factors responsible of activation the senescence-associated secretory phenotypes (SASPs). Consequently, this could enhance the levels of these SASPs factors that will be secreted in the microenvironment promoting malignancy in neighboring cells.

become promoter of tumorigenesis; possibly by activating the senescence-associated secretory phenotypes (SASPs) [39, 40]. Indeed, in the work describing the impact of SASPs on cancer development, the Campisi laboratory showed that while treatment of human prostatic tumor cells with the DNA-damaging agent mitoxantrone (MIT) promote their entry to a senescence state, it also activates SASPs leading to the secretion of promalignant factors such as IL-6 and IL-8 [39]. These two proinflammatory cytokines, are secreted in the microenvironement of senescent cells triggering epithelial-mesenchyme transition and invasiveness, two clear signs of tumor growth and metastasis. Although it is not known whether induction of senescence in the tumor cell itself could enhance their malignant potential at later stages, the activation of 
the SASPs clearly indicates that these cells facilitate the transformation of neighboring cells that are not tumorigenic to become such. Since chemotherapeutic agents normally cause severe stresses, it is possible that some of them trigger the assembly and disassembly of SGs. If the mRNAs encoding SASPs factors are also recruited to these entities, the repeated cycles of translation inhibition/recovery could over time alter their expression pattern causing their massive synthesis and secretion in the surrounding environment. Exploring this possibility and defining whether or not SG assembly/disassembly plays a role in this outcome could help better understand why after being effective at early stages of the treatment some drugs revert and become promoter of malignancy.

\section{ACKNOWLEDGEMENTS}

I am grateful to Dr. S. Di Marco, and Mr. C. von Roretz for critical reading and discussion of the manuscript. I am also grateful to Mr. Xian J. Lian for performing the experiment described in Figure 1 of this manuscript. This work was supported by a CIHR (MOP-67026) and an NCIC (016247) operating grants to I. G. I.G. is a recipient of a TierII Canada Research Chair.

\section{CONFLICT OF INTEREST STATEMENT}

The author of this manuscript has no conflict of interest to declare.

\section{REFERENCES}

1. Campisi J. Aging and cancer cell biology. Aging Cell. 2007; 6:261-263.

2. Schmitt CA. Cellular senescence and cancer treatment. Biochim. Biophys Acta. 2007; 1775:5-20.

3. Hayflick L. The Limited in Vitro Lifetime of Human Diploid Cell Strains. Exp. Cell Res. 1965; 37:614-636.

4. Hayflick L. How and why we age. Exp Gerontol. 1998; 33:639653.

5. Hayflick L. The cell biology of aging. Clin. Geriatr. Med. 1985; 1:15-27.

6. Shamblott MJ, Axelman J, Littlefield JW, Blumenthal PD, Huggins GR, Cui Y, Cheng L, and Gearhart JD. Human embryonic germ cell derivatives express a broad range of developmentally distinct markers and proliferate extensively in vitro. Proc. Natl. Acad. Sci. U S A. 2001; 98:113-118.

7. Campisi J. Senescent cells, tumor suppression, and organismal aging: good citizens, bad neighbors. Cell. 2005; 120:513-522.

8. Campisi J. Aging, tumor suppression and cancer: high wireact! Mech Ageing Dev. 2005; 126:51-58.

9. Kim Sh SH, Kaminker P, and Campisi J. Telomeres, aging and cancer: in search of a happy ending. Oncogene. 2002; 21:503511.

10. Dimri GP. What has senescence got to do with cancer? Cancer Cell. 2005; 7: 505-512.
11. Beausejour CM, Krtolica A, Galimi F, Narita M, Lowe SW, Yaswen $\mathrm{P}$, and Campisi J. Reversal of human cellular senescence: roles of the p53 and p16 pathways. EMBO J. 2003; 22:42124222.

12. Krtolica A and Campisi J. Cancer and aging: a model for the cancer promoting effects of the aging stroma. Int. J. Biochem. Cell Biol. 34, 1401-1414.

13. Krtolica A and Campisi J. Integrating epithelial cancer, aging stroma and cellular senescence. Adv. Gerontol. 2003; 11:109116.

14. Parrinello S, Coppe JP, Krtolica A, and Campisi J. Stromalepithelial interactions in aging and cancer: senescent fibroblasts alter epithelial cell differentiation. J. Cell Sci. 2005; 118:485-496.

15. Brown JP, Wei W, and Sedivy JM. Bypass of senescence after disruption of p21CIP1/WAF1 gene in normal diploid human fibroblasts. Science. 1997; 277:831-834.

16. Campisi J and Yaswen P. Aging and cancer cell biology, 2009. Aging Cell. 2009; 8:221-225.

17. Campisi J. Cellular senescence as a tumor-suppressor mechanism. Trends. Cell Biol. 2001; 11:S27-31.

18. Campisi J. Cancer and aging: yin, yang, and p53. Sci Aging Knowledge Environ. 2002, pe1.

19. Campisi J. Cancer and ageing: rival demons? Nat Rev Cancer. 2003; 3:339-349.

20. Campisi J and d'Adda di Fagagna F. Cellular senescence: when bad things happen to good cells. Nat. Rev. Mol. Cell. Biol. 2007; 8:729-740.

21. Lian XJ and Gallouzi IE. Oxidative Stress Increases the Number of Stress Granules in Senescent Cells and Triggers a Rapid Decrease in p21waf1/cip1 Translation. J. Biol. Chem. 2009; 28:8877-8887.

22. Kedersha NL, Gupta M, Li W, Miller I, and Anderson P. RNAbinding proteins TIA-1 and TIAR link the phosphorylation of elF-2 alpha to the assembly of mammalian stress granules. J. Cell Biol. 1999; 147:1431-1442.

23. Anderson P and Kedersha N. RNA granules. J Cell Biol. 2006 172:803-808.

24. von Roretz C and Gallouzi IE. Decoding ARE-mediated decay: is microRNA part of the equation? J. Cell Biol. 2008; 181:189194.

25. Bruunsgaard $\mathrm{H}$ and Pedersen BK. Special feature for the Olympics: effects of exercise on the immune system: effects of exercise on the immune system in the elderly population. Immunol. Cell Biol. 2000; 78:523-531.

26. Helenius $M$, Makelainen $L$, and Salminen A. Attenuation of NF-kappaB signaling response to UVB light during cellular senescence. Exp. Cell Res. 1999; 248:194-202.

27. Gabai VL and Sherman MY. Invited review: Interplay between molecular chaperones and signaling pathways in survival of heat shock. J. Appl. Physiol. 2002; 92:1743-1748.

28. Kregel KC, Moseley PL, Skidmore R, Gutierrez JA, and Guerriero V Jr. HSP70 accumulation in tissues of heat-stressed rats is blunted with advancing age. J. Appl. Physiol. 1995; 79:1673-1678.

29. Luce $M C$ and Cristofalo VJ. Reduction in heat shock gene expression correlates with increased thermosensitivity in senescent human fibroblasts. Exp. Cell Res. 1992; 202:9-16.

30. Effros RB, Zhu $X$, and Walford RL. Stress response of senescent T lymphocytes: reduced hsp70 is independent of the proliferative block. J. Gerontol. 1994; 49:B65-70. 
31. Mazroui R, Di Marco S, Kaufman RJ, and Gallouzi IE. Inhibition of the ubiquitin-proteasome system induces stress granule formation. Mol. Biol. Cell. 2007; 18:2603-2618.

32. Anderson $P$ and Kedersha N. Stress granules: the Tao of RNA triage. Trends Biochem. Sci. 2008; 33:141-150.

33. Kedersha $\mathrm{N}$ and Anderson P. Stress granules: sites of mRNA triage that regulate mRNA stability and translatability. Biochem. Soc. Trans. 2002; 30:963-969.

34. Mazroui R, Sukarieh R, Bordeleau ME, Kaufman RJ, Northcote P, Tanaka J, Gallouzi I, and Pelletier J. Inhibition of ribosome recruitment induces stress granule formation independently of eukaryotic initiation factor 2alpha phosphorylation. Mol. Biol. Cell. 2006; 17:4212-4219.

35. Dang Y, Kedersha N, Low WK, Romo D, Gorospe M, Kaufman $\mathrm{R}$, Anderson P, and Liu JO. Eukaryotic initiation factor 2alphaindependent pathway of stress granule induction by the natural product pateamine A. J. Biol. Chem. 2006; 281:32870-32878.

36. Marusyk A and DeGregori J. Replicational stress selects for p53 mutation. Cell Cycle. 2007; 6:2148-2151.

37. Marusyk A, Wheeler L, Mathews CK, and DeGregori J. p53 mediates senescence-like arrest induced by chronic replicational stress. Mol. Cell Biol. 2007; 27:5336-5351.

38. Collado M, Blasco MA, and Serrano M. Cellular senescence in cancer and aging. Cell. 2007; 130:223-233.

39. Coppe JP, Patil CK, Rodier F, Sun Y, Munoz DP, Goldstein J, Nelson PS, Desprez PY, and Campisi J. Senescence-associated secretory phenotypes reveal cell-nonautonomous functions of oncogenic RAS and the p53 tumor suppressor. PLoS. Biol. 2008; 6:2853-2868.

40. Rodier F, Coppe JP, Patil CK, Hoeijmakers WA, Munoz DP, Raza SR, Freund A, Campeau E, Davalos AR, and Campisi J. Persistent DNA damage signalling triggers senescenceassociated inflammatory cytokine secretion. Nat. Cell Biol. 2009; 11:973-979. 